\title{
Adjustable Quarto Stimuli (T, pH, Redox, Hyperthermia) Targeted Nanocontainers (Nano4Dox and Nano4Cis) for Cancer Therapy Based on Trojan Horse Approach
}

\author{
George Kordas* \\ Institute of Nanoscience and Nanotechnology, Greece
}

*Corresponding author: George Kordas, Institute of Nanoscience and Nanotechnology, NCSR Demokritos, 15310 Ag Paraskevi Attikis, Greece.

\author{
Received Date: July 05, 2018 \\ Published Date: July 20, 2018
}

\begin{abstract}
We developed a quadrupole stimuli responsive targeted nanomedicine platform with the key goal to provide a solution to the outstanding problem "How can the delivered therapeutic dose to the tumour be increased while at the same time reducing the dose in healthy tissue?" When accomplishing this goal, we will offer a huge improvement in the length and quality of life of cancer patients. Our preliminary animal study give hope in that course. We attempted to accomplish this goal via an innovative and patented therapeutic approach based on novel polymeric nanocarriers (NCs), which enables targeted drug delivery in tumors. The in-vivo results suggest that both Nano4Dox and Nano4Cis platforms outperforms the commercial standards like Doxil@ and Lipoplatin, respectively. These platforms offer a significant business opportunity besides the therapeutic value offering to millions of people hope for better life and cure.
\end{abstract}

\section{Introduction}

In clinical research, most commercial drugs fail to accomplish therapy due to intolerable adverse effects and limited efficacy. The latter is attributed to the limited drug quantity that reaches tumors and a large portion of the remaining drug that affects healthy tissue. The resulting attrition rate in pharmaceutical development is one of the main causes of industrial losses and high prices in e.g. oncology medicine [1]. Due to similar safety concerns, many marketed treatments are administered at suboptimal doses and do not exert their full effect. To this situation, there is a pressing demand for (1) strategies to effectively access tumors with high therapeutic potential at sufficient drug concentrations, and (2) ways to elevate the efficiency of existing and developmental drugs to safely target a range of diseases.

The encapsulation of compounds in nanocarriers (NC) has emerged as one of the most promising approaches to significantly improve local bioavailability. These NC protect the drug (e.g. nucleotides) against degradation and protect healthy tissues against toxic effects (e.g. toxic anticancer drugs). Despite this promise, the number of nanocarrier-based treatments that have reached the market is still disappointing. The main problem in the development of these carriers is the need for sufficient stability and robustness when passing through the bloodstream, while being capable of unloading the drug when the target tissue has been reached. This trade-off remains a significant challenge in the development of nanocarriers.

Here, we present the quadrupole stimuli targeted nanocontainers platform that can be loaded with any commercial drug thus alleviating the above-mentioned drawbacks of chemotherapy. The idea behind the present platform was reported several times last year's where we describe the manufacturing several times [2-19] that show high promise to address this issue. It was shown that loading these nanocontainers with extremely toxic commercial drugs into the so called Nano4XX (e.g. doxorubicin, leuprolide, daunorubicin, etc.) loose toxicity and increase therapeutic effect. Our Nano4XX platforms are engineered from biocompatible and biodegradable polymers and are unique in (1) their stability and resistance to drug leakage in the circulation, while (2) they exert conformational changes under specific conditions 
targeting pathological tissue, inducing localised drug release. This technology leverages the fact that tumors (as well as other types of diseased tissue) are known to have specific extracellular environments with lower $\mathrm{pH}$, higher temperature and/ or enhanced glutathione levels compared to healthy tissues. Furthermore, the Nano4XX platforms are grafted with targeting groups e.g. folic acid for breast cancer, leuprolide for prostate cancer, etc. The Nano4XX platforms are designed such to become unstable specifically under the conditions present in cancer, the release of the encapsulated compounds is directed at the target tissue. As such, encapsulating drugs in our Nano4XX has a beneficial effect on the accumulating dose in target tissue - and thus the intended effect of the drug as well as on unwanted side-effects on healthy tissues. The Nano4XX platforms integrate four stimuli, namely $\mathrm{pH}$, temperature (T), reducing environments (glutathione) and alternating magnetic fields (Figure 1). Loading and release studies with anthracycline antibiotics confirm the high loading capacity of the NCs along with the sensitive response to any needed combination of stimuli.

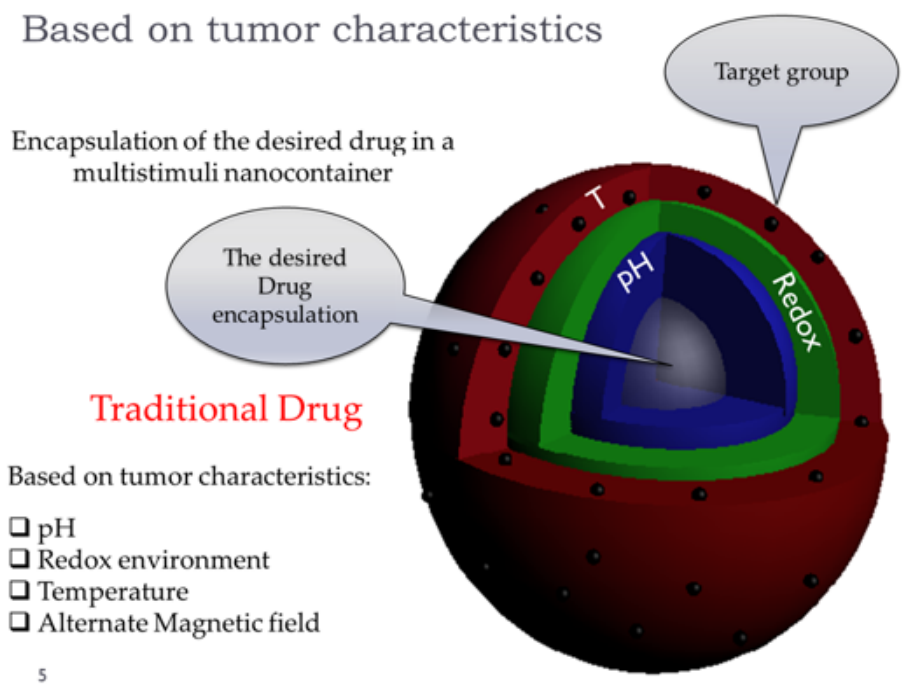

Figure 1: Principle of the Nano4XX platform.

We have observed in vivo and in vitro evidence of the functional added value of our proprietary [20] Nano4XX for the delivery of anti-tumour and antibacterial drugs. Furthermore, we have a strong IP position and developed a business plan that describes the optimal route-to-market for our technology [21]. Just to mention the business opportunity of our system, the doxorubicin global market volume was USD 0.81 billion in 2015 and is expected to reach a value of USD 1.38 billion by 2024, according to a new report by Grand View Research, Inc. [22]. If our system passes clinical trial, we will provide first a safe delivery of drugs to tumors and second a great business opportunity. Because we can load in our Nano4XX platform any commercial drug, our technology presents the business potential accordingly multiplied by the market value of each drug. A business revolution in cancer therapy.

\section{Experimental Design}

The previous publications [2-19] and the patent [20] describes thoroughly the manufacturing procedures of our Nano4XX platforms. The manufacturing involves several steps. The first step is the production of a spherical Poly (Methyl Methacrylate) template. The second step involves coating of the template with monomers that are thermo-, $\mathrm{pH}$ - and redox sensitivity, like Dimethyl Amino Ethyl Methacrylate (DMAEMA), Acrylic Acid (AA) and $\mathrm{N}, \mathrm{N}^{\prime}$-(disulfanediylbis(ethane-2,1-diyl))bis(2-methylacrylamide) (Disulfide or DS), respectively. The third step consists of removing the template to leave behind a hole that is filled by commercial drugs. In the present study, we filled the nanocontainers with doxorubicin and cis-platin. The surface of the Nano4(Dox and Cis) platforms was functionalized with magnetite nanoparticles to establish sensitivity in external alternating magnetic field (AMF). Furthermore, we grafted the Nano4XX platforms with targeting groups like folic acid and leuprolide to target breast and prostate cancer, respectively. Hyperthermia rises the temperature of the final Nano4XX, another stimulus. This way, we witness the Nano4XX (Dox, Cis) platforms. In our proof of concept, the Nano4XX (Dox, Cis) platforms were associated with the commercial analogs like Doxil and Lipoplatin. Radiolabeling of the Nano4XX platforms with $99 \mathrm{mTc}$ intravenously injected on female normal swiss mice determines their biodistribution in presence or not of targeting groups. Targeting of our Nano4XX platforms was established both in-vivo and in-vitro. We observed that the Nano4XX platforms outperform the commercial drugs like Doxil and Lipoplatin present a better therapeutic result but also an incredible business prospect. The Nano4XX platform will lead to a tremendous industrial application and beyond that will boost nanotechnology.

\section{In Vitro and In Vitro Evidence of Targeting Cancer}

Our innovative multi-stimuli-controlled release system is capable to stay robust in the bloodstream, while allowing drug release upon triggering by the proper internal (e.g. $\mathrm{pH}$ and glutathione) or external (e.g. magnetism and temperature) stimuli when the target cells have been reached. Another huge advantage of our NCs is the ability to functionalize their surface. 'Stealth' inducing molecules can improve their circulation time in blood (e.g. PEG), while small molecules (e.g. Folic Acid, VGFR analogues) or peptides (e.g. GFLG, Leuprolide analogues and cRGD) can be used 
as targeting moieties. For example, folic acid is a small organic molecule which receptor is overexpressed at the surface of specific hormone depended cancer cells. Folic acid functionalized NCs are shown to localize at cervical cancer cells (HeLa) (Figure 2, right) in contrast to non-targeted NCs (Figure 2, left). After binding to the folate receptor, the cell membrane will fold inside, where a localised drug release is triggered. One perceives from this figure, that when the platform Nano4XX is not grafted with folic acid the nanocontainers agglomerate outside cancer cells. Instead, grafting the nanocontainers with folic acid induces incorporation into the cell and drug release occurs. Figure 3 suggests the mechanism of intercalation, drug release into the tumor cells and therapy of cancer.
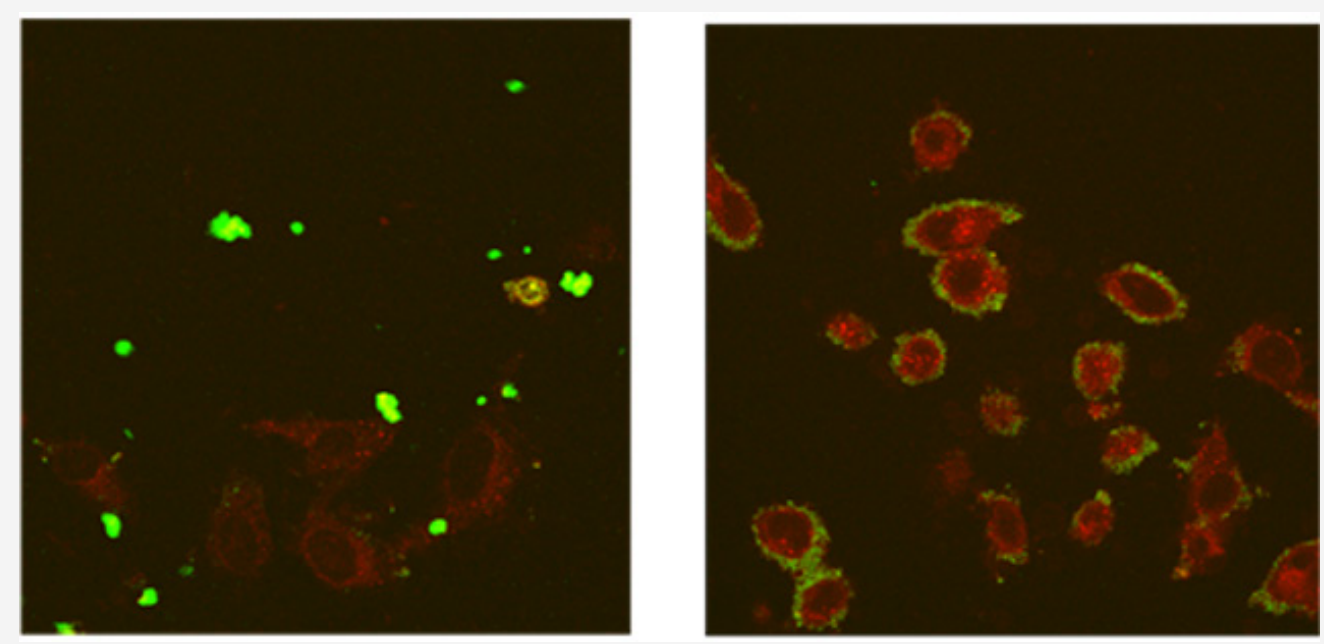

Figure 2: Targeted internalization by attaching folic acid to our NCs. On the left NCs are seen dispersed outside HeLa cells, while on the right targeted NCs are incorporated in the cells.

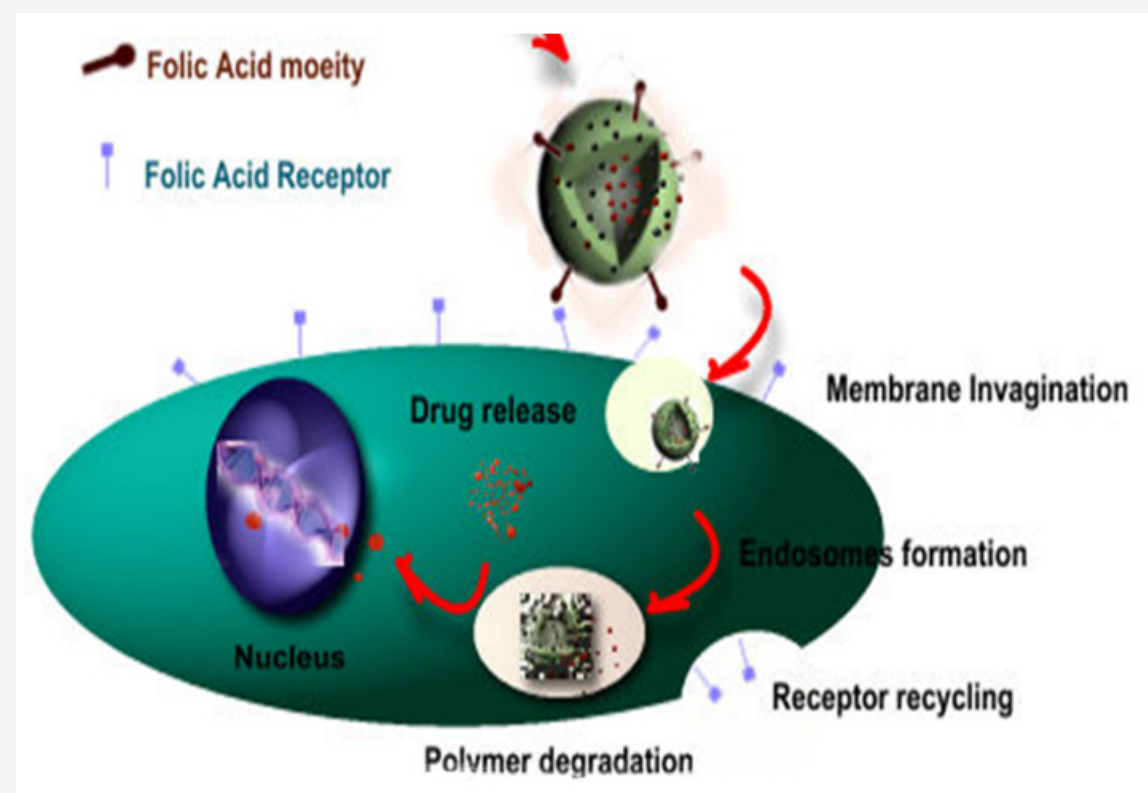

Figure 3: Internalization of the Nano4Dox into cancer cell.

The targeting ability of NCs with folic acid has also been validated in mice xenografts with dynamic in vivo imaging. Based on our preliminary animal studies we have promising results about their toxicity, biodistribution and pharmacokinetics. Targeting was observed after grafting the nanocontainers with gamma emitting radionuclide $(99 \mathrm{mTc})$ detected by PET. Figure 4 shows PET results in tumour for nanocontainers with and without grafting with folic acid in various organs. In case of liver, the accumulation of the Nano4Dox platforms amounts to $45 \%$ and $24 \%$ without and with grafting folic acid, respectively. The concentration of the nanocontainers without targeting groups in the tumour is neglectful as can be obtained in the same figure. Contrary, the concentration of the nanocontainers in cancer is about $5 \%$ after one hour of accumulation. This is a clear evidence that the folic acid grafted Nano4Dox platform is attached to tumor confirming the invitro experiments of Figure 4. The question is whether there is a significant therapeutic effect after delivering the Nano4XX platform into the blood stream. This has been tested in two cases like the Nano4Dox and Nano4Cis platforms. 


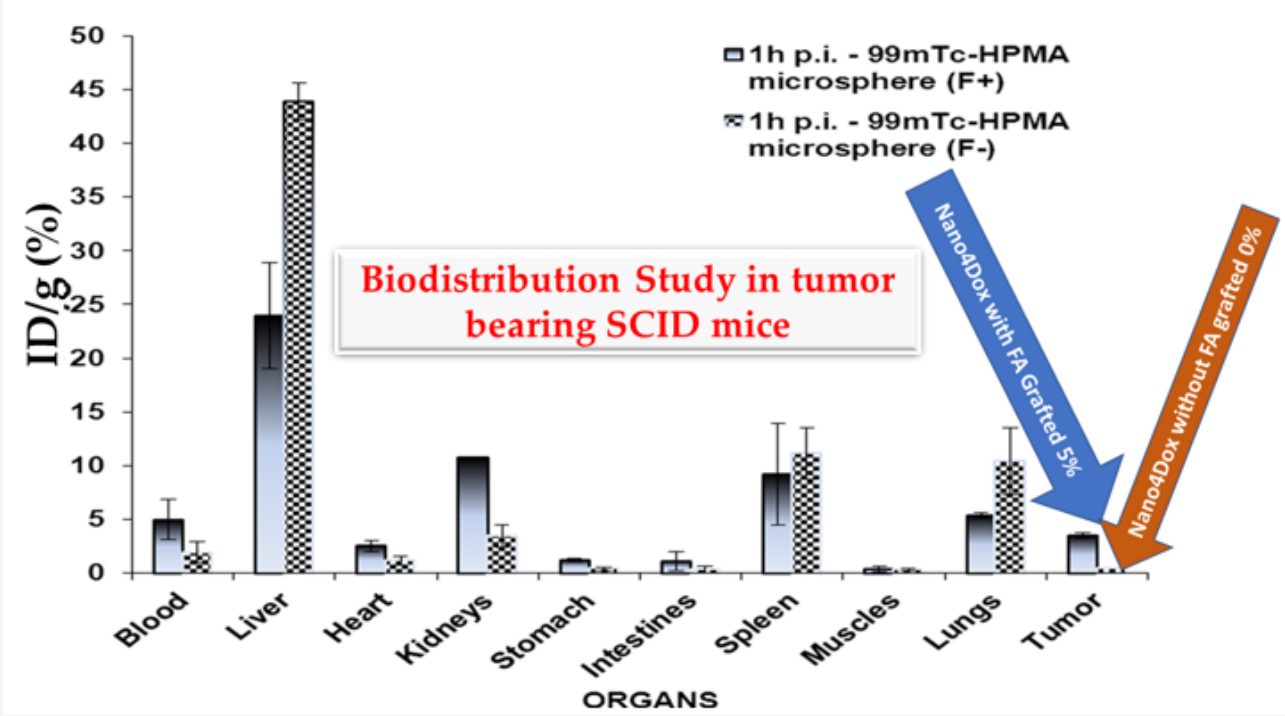

Figure 4: Distribution of the Nano4Dox in various organs.

\section{Therapeutic Efficacy}

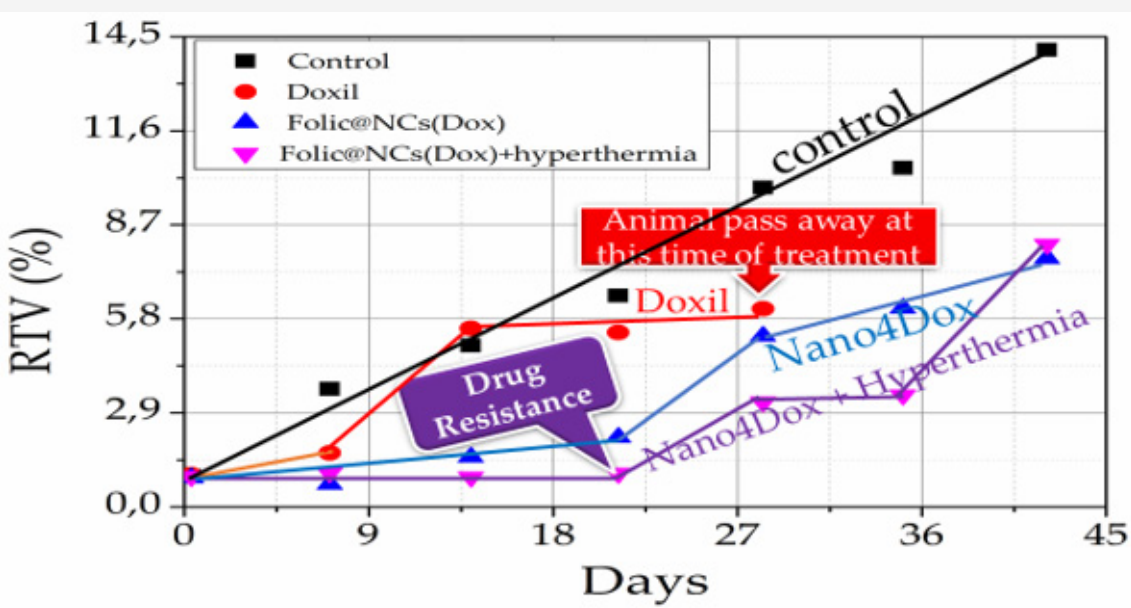

Figure 5: Tumor efficacy experiment in different groups. Group 1: Control group injected with PBS (Black), Group 2: Nano4Dox (Red), Group 3: Nano4Dox (Blue) combined with 15 min hyperthermia treatment, Group 4: Doxil@ injected (Violet).

The main goal of the work was concerned to determine whether the Nano4XX platforms perform better than the commercial drugs, like Doxil $®$ and Lipoplatin, to start the comparison. First, we will discuss results of the comparison between Doxil@ and Nano4Dox. The therapeutic efficacy study was performed using HeLa cervical tumor bearing SCID mice and measuring the cancer volume as a function of the time after delivering the targeted Nano4Dox platform and Doxil@. The tumor efficacy experiments have been performed in 4 different groups by tumor bearing SCID mice. Each group has 6 repetitions. The first group was the control group, the second group was treated with the targeted Nano4Dox platform and third (blue line) group was treated again with the targeted Nano4Dox platform using hyperthermia and final the gold standard nanotechnology product Doxil@. The tumor volume was determined by using an automatic caliper. The treatment and tumor measurements were performed each week for a duration of 45 days. Figure 5 shows the results of these experiments. The blue triangles present the growth of cancer as a function of time in the SCID mice treated with
Nano4Dox without grafting with folic acid. The black rectangles are attributed to the experiment of the animals treated with doxorubicin. This plot shows an increase of cancer volume with the time. The red circles present the treatment with Doxil@. One can observe an increase of cancer volume with the time, though the experiment stops at 26 days due to the death of the animals. Opposing to this result, we observe a $20 \%$ volume reduction in twenty days (blue triangles) after delivering Nano4Dox grafted with folic acid. This beneficial therapeutic outcome improves when hyperthermia is applied during therapy (violet triangles) leading to a better reduction of cancer volume as a function of the time. Obviously, our folic acid grafted Nano4Dox platform outperforms the existing carrier technology (liposomes, dendritic polymers, polymeric hydrogels, micelles, etc.) and yields to an outstanding therapeutic effect. The Nano4Dox platform establishes a significant improvement over the state of the art, since it is the first to integrate four stimuli $(\mathrm{pH}$, temperature, reducing environments and alternating magnetic fields) as well as proprietary targeting 
capabilities. The "active targeting" aspect of our NCs results from the surface attachment of certain ligands that bind to proteins overexpressed on tumor cells, which has been shown to improve the target specificity and improve therapeutic activity. Due to this targeting, $5 \%$ of the compound reaches the tumor; this is absolute best in class. The above result supports that targeted Nano4Dox platform exhibits better tumor efficiency than the gold standard Doxil ${ }^{\circledR}$ an absolute blockbuster nanomedicine in oncology.

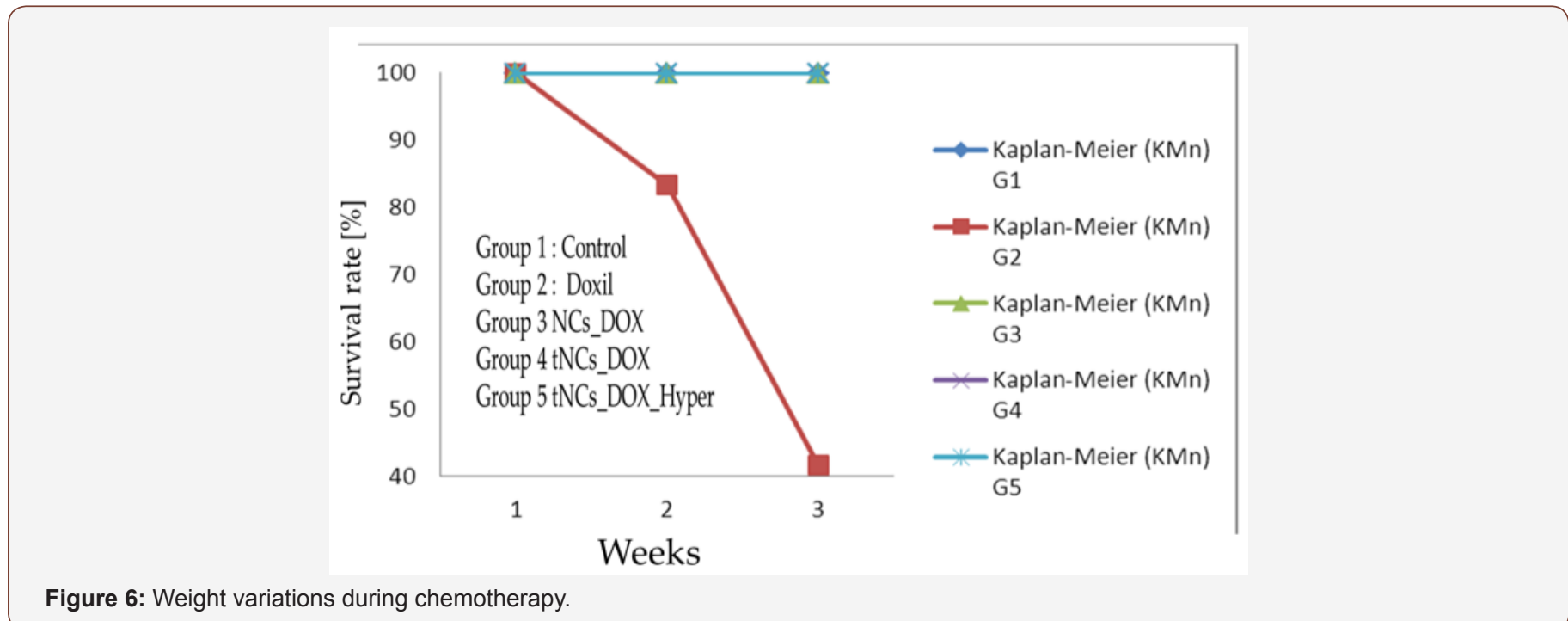

Figure 6 shows also that animal treated with Doxil@ pass away after three weeks of treatment (red line). In more extensive study, Fig. 6 presents the animal survival rate treated as described in the experiments of Fig. 5. As all animals are reduced with the treatment of Doxil $®$ where all pass away after three weeks, the animal treated

with our Nano4Dox platform survive the treatment. An amazing result encourages work with our delivery system. The experiment is stopped after 43 days for ethical reasons. Though, the hyperthermia treated animal delivered the folic acid targeted Nano4Dox show drug resistance phenomenon after 20 days of treatment.

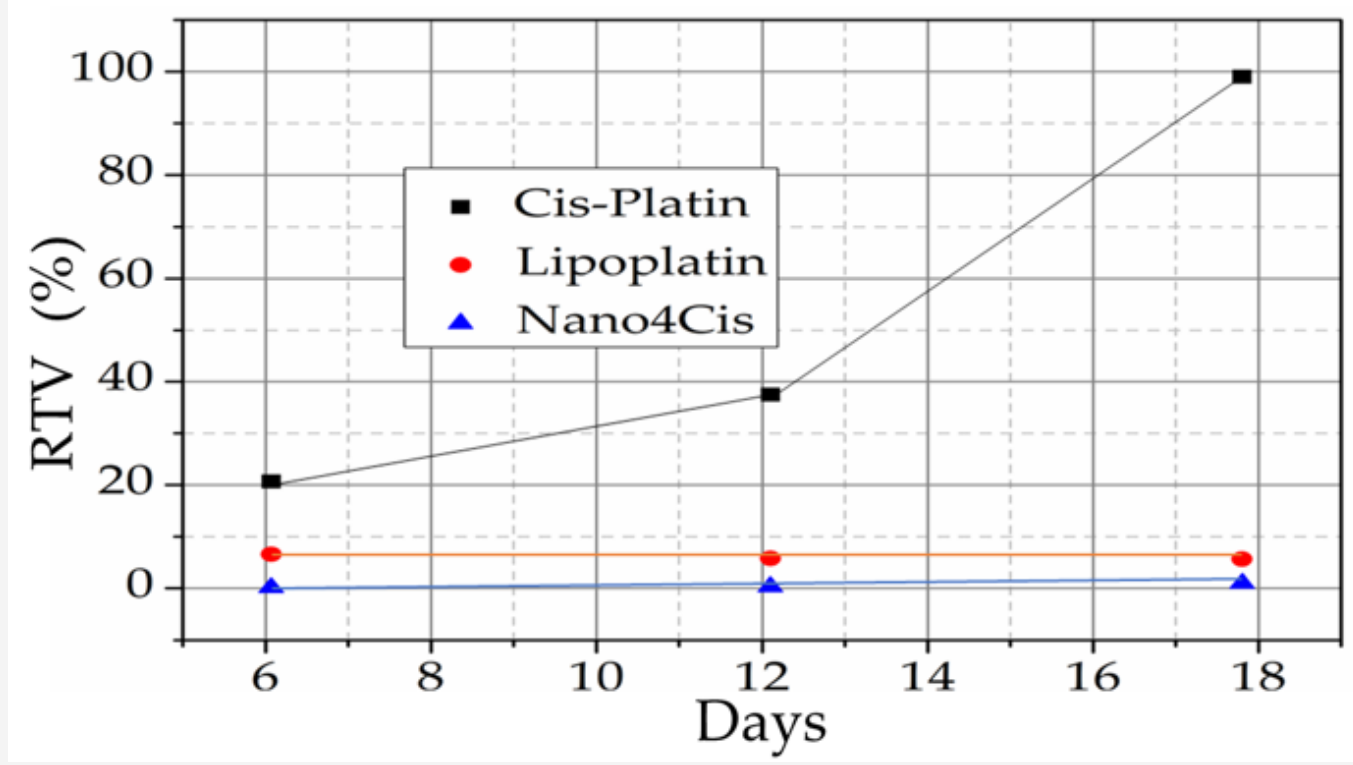

Figure 7: Cancer volume as a function of time after delivering Cis-platin (black), Lipoplatin (red) and Nano4Cis (blue).

The treatment efficacy was extended in the Nano4Cis platform that was compared with Lipoplatin. Cis-platin that is a traditional chemotherapeutic drug but presents many side effects such as hematotoxicity and other significant side effects leading to the treatment limitations for cancer therapy. Cis-platin loaded in multi stimuli targeted Nano4Cis platform do not exhibit the known side effects of Cis-platin. In detail the drug was loaded on our targeted Nano4Cis by using PBS at $\mathrm{pH}=7.4$ for 24 hours treatment. The mixture was centrifuged and free cis platin was determined on supernatant. The loading capacity was determined by using the standard curve method and calculations depicts that the LC \% (loading capacity) was $82 \%$ and was EE \% (encapsulation efficacy) $=92 \%$. We compare in vivo Lipoplatin with our folic acid targeted Nano4Cis platform. For this evaluation was used tumor bearing animals by HeLa cells for tumor efficacy investigation. We used 3 group of animals. The first was the control group, the second 
was the lipoplatin and the third was Nano4Cis platform. The nanocontainers were injected in SCID mice. The studies measure the tumor volume as a function of the time. Figure 7 demonstrates the results of our study by which we can strongly support that our targeted nano4Cis platform behave better than the lipoplatin.

\section{Pharmacokinetic Studies}

Pharmacokinetic study of the Nano4Dox platforms were performed in CD1 mice. Animals were dosed intravenously
(IV) with $5 \mathrm{mg} / \mathrm{kg}$ (in reference to Dox) injections of Nano4Dox and free Dox in saline. Each point represents four animals. The analysis was performed by using a calibrated LC-MS/MS. In this work, we analyzed plasma, lung, liver, and kidney samples after homogenization. Figure 8 shows the results of this work. In plasma, the doxorubicin concentration is below $25 \mathrm{ng} / \mathrm{ml}$ and goes down to zero in 50 hours. Similar steady state was observed in the liver, much lower doxorubicin concentration than free doxorubicin. Different behavior was observed for lungs and kidneys.
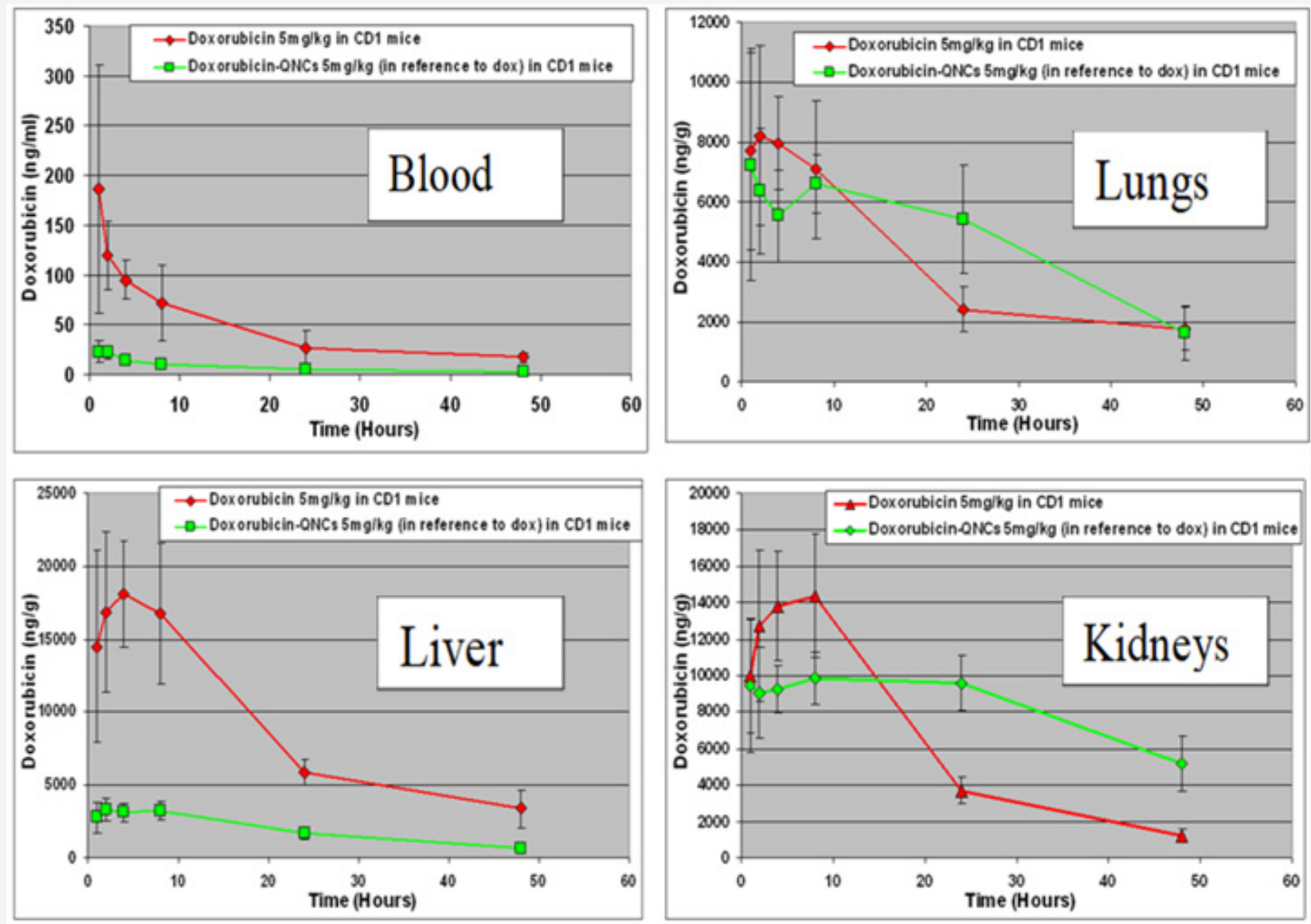

Figure 8: Pharmacokinetics of Quadrupole NCs in CD1 mice in different organs.

The results of this study where the biodistribution of Nano4Dox and free Dox was determined in various organs suggests that we have reduced systemic circulation of Dox, possibly could result to reduced peripheral toxicity. Supplementary, the significantly lower levels of Dox in the kidneys which reduced nephrotoxicity which is a major issue for anthracyclines. It is worth mentioning that observed lower Cmax in all the examined tissues in comparison to the Cmax following the administration of Dox leading to less peripheral toxicity. More sustained levels of Dox in liver and lungs enhancing the efficacy if targeting these tissues. Eventually no significant acute toxicities were observed demonstrating that the Nano4Dox can be a promising smart carrier. A complete in vivo toxicity study of quatro stimuli Nanocontainers in pregnant rats has been published recently [23].

\section{Conclusion}

Polymeric NCs present many advantages compared to other drug delivery systems. A characteristic example is their comparison with liposomes, which are currently used to deliver doxorubicin. Polymeric NCs have much better shelf stability, are less easily oxidized in contrast to liposomes due to their liposomal phospholipids and have fewer limitations on the drug loading spectra and volume. Another unique potential of our nanoparticle carriers is their ability to encapsulate and deliver poorly soluble cancer therapeutics, such as the taxanes. The Nano4XX platforms may some day be loaded with extremely toxic drugs to treat cancer more efficiently than any other way of treatment.

\section{Acknowledgements}

The work is conducted under support of the European Research Council (ERC) under the IDEAS program under contracts:

1. Advanced Grant: "A Novel Nanocontainer drug carrier for targeted treatment of prostate cancer "Nanotherapy" Nos. 232959, and

2. Proof of Concept Grant: Nos. 620238. 
The financial support is greatly appreciated.

The assistance of the collaborators is also respected: P. Bilalis, C. Tapeinos, G. Pappas, A. Chatzipavlidis, E.K. Efthimiadou, A.F. Metaxa, G. Loudos, E. Fragogeorgi, P. Kainourgios, N. Kostomitsopoulos, P. Lelovas, C. Tamvakopoulos and N. Boukos.

\section{References}

1. Pammolli F, Magazzini L, Riccaboni M (2011) The productivity crisis in pharmaceutical R\&D. Nat Rev Drug Discov 10(6): 428-438.

2. Efthimiadou EK, Tapeinos C, Bilalis P, Kordas G (2011) New approach in synthesis, characterization and release study of $\mathrm{pH}$-sensitive polymeric micelles, based on PLA-Lys-b-PEGm, conjugated with doxorubicin. Journal of Nanoparticle Research 13(12): 6725-6736.

3. Chatzipavlidis A, Bilalis P, Efthimiadou EK, Boukos K, Kordas G (2011) Sacrificial Template-Directed Fabrication of Superparamagnetic Polymer Microcontainers for $\mathrm{pH}$-Activated Controlled Release of Daunorubicin Langmuir 27(13): 8478-8485.

4. Efthimiadou EK, Tziveleka LA, Bilalis P, Kordas G (2012) Novel PLA modification of organic microcontainers based on ring opening polymerization: synthesis, characterization, biocompatibility and drug loading/release properties. International Journal of Pharmaceutics 428(1-2):134-142.

5. Metaxa AF, Efthimiadou EK, Boukos N, Kordas G (2012) Polysaccharides as a source of advanced materials: Cellulose hollow microspheres for drug delivery in cancer therapy. Journal of Colloid and Interface Science 384(1): 198-206.

6. Bilalis P, Efthimiadou EK, Chatzipavlidis A, Boukos N, Kordas G (2012) Multi-responsive polymeric microcontainers for potential biomedical applications: synthesis and functionality evaluation. Polymer International 61( 6): 888-894.

7. Angelopoulou A, Efthimiadou EK, Kordas G (2012) Dextran modified $\mathrm{pH}$ sensitive silica hydro-xerogels as promising drug delivery scaffolds. Materials Letters 74: 50-53.

8. Tapeinos C, Efthimiadou EK, Boukos N, Koklioti A, Charitidis C, et al (2013) Design strategies for fluorescent biodegradable polymeric biomaterials. Journal of Materials Chemistry B 1(2): 194-203.

9. Kainourgios P, Efthimiadou EK, Tziveleka LA, Pappas GS, Boukos N, et al. (2013) Comparative study of LbL and crosslinked pH sensitive PEGylated LbL microspheres: Synthesis, characterization and biological evaluation. Colloids and Surfaces B: Biointerfaces 104: 91-98.

10. Chatzipavlidis A, Bilalis P, Tziveleka LA, Boukos N, Charitidis C, et al. (2013) Nanostructuring the surface of dual responsive hollow polymer microspheres for versatile utilization in nanomedicine-related applications. Langmuir 29(30): 9562-9572.
11. Efthimiadou EK, Tapeinos C, Chatzipavlidis A, Boukos N, Fragogeorgi E, et al. (2014) Dynamic in vivo imaging of dual-triggered microspheres for sustained release applications: Synthesis, characterization and cytotoxicity study. International Journal of Pharmaceutics 461(1-2): 54 63.

12. Efthimiadou EK, Tapeinos C, Tziveleka LA, Boukos N, Kordas G (2014) $\mathrm{pH}-$ and thermo-responsive microcontainers as potential drug delivery systems: Morphological characteristic, release and cytotoxicity studies. Materials Science and Engineering C 37: 271-277.

13. Tziveleka LA, Bilalis P, Chatzipavlidis A, Boukos N, Kordas G (2014) Development of multiple stimuli responsive magnetic polymer nanocontainers as efficient drug delivery systems. Macromolecular Bioscience 14(1): 131-141.

14. Angelopoulou A, Efthimiadou EK, Boukos N, Kordas G (2014) A new approach for the one-step synthesis of bioactive PS vs. PMMA silica hybrid microspheres as potential drug delivery systems. Colloids and Surfaces B: Biointerfaces 117: 322-329.

15. Metaxa AF, Efthimiadou EK, Boukos N, Fragogeorgi EA, Loudos G, et al. (2014) Hollow microspheres based on-folic acid modifiedhydroxypropyl cellulose and synthetic multi-responsive bio-copolymer for targeted cancer therapy: controlled release of daunorubicin, in vitro and in vivo studies. Journal of Colloid and Interface Science 435: 171181.

16. Papadopoulos C, Loudos G, Efthimiadou EK, Kordas G, Kagadis G (2016) Numerical simulation and experimental validation of magnetic nanoparticle hyperthermia. Physica Medica: European Journal of Medical Physics 32(3): 259.

17. Rouchota M, Loudos G, Efthimiadou EK, Kordas G, Kagadis GC (2016) Assessment of low energy X-ray imaging for magnetic and gold nanoparticles. Physica Medica: European Journal of Medical 32(3): 257.

18. Tapeinos C, Efthimiadou EK, Boukos N, Kordas G (2016) Sustained release profile of quatro stimuli nanocontainers as a multi sensitive vehicle exploiting cancer characteristics. Colloids and Surfaces B: Biointerfaces 148: 95-103.

19. Efthimiadou EK, Fragogeorgi E, Palamaris L, Karampelas T, Lelovas P, et al. (2017) Versatile quarto stimuli nanostructure based on trojan horse approach for cancer therapy: synthesis, characterization, in vitro and in vivo studies. Materials Science and Engineering: C 79(1): 605-612.

20. Kordas G, Efthimiadou EK (2016) Nanotechnology in Cancer Treatment as a Trojan horse: from the Workbench in Pharmaceutical Industry. UP Publications 1(1): 1-15.

21. Kordas G (2016) Business plan of nano4 Chem.

22.https: / /www.grandviewresearch.com/press-release/globaldoxorubicin-market

23. Lelovas P, Efthimiadou EK, Mantziaras G, Siskos N, Kordas G, et al. (2018) Regulatory Toxicology and Pharmacology, Elsevier, USA. 